\title{
Opportunistic Multiple Access for Cognitive Radio Networks
}

\author{
Amr A. El-Sherif, Ahmed K. Sadek, and K. J. Ray Liu
}

\begin{abstract}
In this paper, opportunistic multiple access to the under-utilized channel resources is investigated. Exploiting source burstiness, secondary cognitive nodes utilizes primary nodes' periods of silence to access the channel and transmit their packets. Cognitive relays could also make use of these silence periods to offer spatial diversity without incurring bandwidth efficiency losses. First, we consider the cognitive cooperation protocol and propose two different relay assignment schemes. Comparison between the proposed schemes is carried out through a maximum stable throughput analysis of the network. Then, secondary nodes access to the remaining idle channel resources is investigated. Queueing theoretical analysis and numerical results reveal that despite the fact that relays occupy part of the idle resources to provide cooperation, secondary nodes surprisingly achieve higher throughput in the presence of relays. The rationale is that relays help primary nodes empty their queues at faster rates, therefore, secondary nodes observe increased access opportunities to the channel.
\end{abstract}

Index Terms-Cooperative communications, cognitive radio, queuing theory, multiple access.

\section{INTRODUCTION}

T HE SCARCITY of energy and bandwidth, the two fundamental resources for communications, imposes severe limitations on the development of communications networks in terms of capacity and performance. Among the new technologies that have emerged recently in the effort to intelligently and efficiently utilize these scarce resources are cooperative communications and cognitive spectrum sharing. Both technologies have shown great potential for enhancing the performance of wireless networks and meeting the demands of future wireless applications.

In cooperative communications [1]-[3], portion of the channel resources are assigned to one or more relays for cooperation. These relays cooperate with a source node to help in forwarding its data to a destination. This can achieve spatial diversity as the data is transmitted via spatially independent channels, but also results in some bandwidth efficiency loss because of the channel resources assigned to the relays to perform their task. In [4], the authors developed a cognitive multiple access protocol that overcomes the problem of bandwidth efficiency loss. The protocol in [4] exploits source burstiness to enable cooperation during silence periods

Manuscript received 30 November 2009; revised 24 May 2010.

A. A. El-Sherif is with the Department of Electrical Engineering, Alexandria University, Alexandria 21544, Egypt (e-mail: amr.elsherif@ieee.org).

A. K. Sadek is with Corporate R\&D Qualcomm Inc., San Diego, CA 92121, USA (e-mail: asadek@qualcomm.com).

K. J. R. Liu is with the Department of Electrical and Computer Engineering, University of Maryland, College Park, MD 20742, USA (e-mail: kjrliu@umd.edu).

Digital Object Identifier 10.1109/JSAC.2011.110404. of different nodes in a TDMA network. In other words, a cooperative relay will detect and utilize empty time slots in the TDMA frame to retransmit failed packets. Therefore, no extra channel resources are allocated for cooperation and the system encounters no bandwidth losses. The authors analyzed the protocol's performance from a maximum stable throughput point of view, and their results revealed significant performance gains over conventional cooperation strategies.

The second promising technology aiming at better utilization of the available channel resources is cognitive radio [5]. Cognitive radio prescribes the coexistence of licensed (or primary) and unlicensed (secondary or cognitive) radio nodes on the same bandwidth. While the first group is allowed to access the spectrum any time, the second seeks opportunities for transmission by exploiting the idle periods of primary nodes. In [6] and [7] the cognitive radio problem was investigated from an information theoretic standpoint, where the cognitive transmitter is assumed to transmit at the same time and on the same bandwidth of the primary link, being able to mitigate its interference toward the primary through complex precoding techniques that are based on the perfect prior information about the signal transmitted by the primary. Centralized and decentralized protocols at the media access control (MAC) layer aiming at minimizing secondary nodes interference with primary transmissions have been studied in [8] and [9] by modeling the radio channel as either busy (i.e., the primary user is active) or available (i.e., the primary user is idle) according to a Markov chain.

It is clear that cooperative communications and cognitive radios are closely related problems in the sense that the available unused channel resources can be utilized to improve the primary system performance via cooperation, or it can be shared by a secondary system to transmit new information. Despite this fact, these two problems have been studied independently. In this paper, we address the issue of exploiting the underutilized channel resources by both cognitive cooperative relays and cognitive secondary nodes. Our main focus is on how this coexistence of primary relays and secondary nodes affects the performance of both primary and secondary networks. At a first glance one might jump to the conclusion that since relays are part of the primary network thus having higher priority over secondary nodes, then the primary network will benefit from cooperation while secondary nodes will suffer from reduced channel access opportunities. We Will prove that this argument is not correct, and that even in the situation of interfering relays and secondary transmissions, both networks will benefit from the presence of relays in terms of maximum stable throughput. 
First, we consider the uplink of a TDMA network as the primary network, and study how cognitive relays can exploit the empty time slots to offer help to the primary nodes. In [4], the authors have studied this problem in a network with single relay. Here we consider the effect of multiple relays and address the issue of how relays share the empty time slots among themselves. Furthermore, two relay selection criteria are presented, namely, the nearest neighbor and the maximum success probability, their performance in terms of maximum stable throughput is thoroughly investigated. Then we consider the issue of secondary nodes also trying to exploit empty time slots in the primary network. While most of the research on cognitive radios has focused on the dynamic spectrum sharing aspect of the problem, we focus on the opportunistic multiple access aspect of the problem in the TDMA framework.

To gain access to the channel, secondary nodes sense the channel for primary activity. In order to have an upper and lower bound on the system's performance, we study two different scenarios. The first is when secondary nodes have the ability to perfectly sense relays transmissions, and thus access the channel when all primary nodes and relay nodes queues are empty. In the second scenario secondary nodes cannot sense relays transmissions at all. Since the cognitive principle is based on the idea that the presence of the secondary system should be transparent to the primary system, appropriate countermeasures should be adopted at the secondary nodes to minimize interference with relay transmissions. The stability region is characterized for these two scenarios and compared to the case where the primary network doesn't employ relays. Analytical and numerical results reveal that although relays occupy part of the empty time slots that would have been available to secondary nodes, it is always beneficial to both primary and secondary nodes that the maximum possible number of relays be employed. On one hand, relays help the primary network achieve higher stable throughput by offering different reliable paths for the packets to reach the destination. On the other hand, relays will help primary nodes empty their queues at a much faster rates, thus providing secondary nodes with more opportunities to transmit their own information.

At this point we need to emphasize on the practical importance of the work presented in this paper. As discussed above our model deals with the uplink of a TDMA network. TDMA is widely used in many networks such as the GSM cellular networks, Bluetooth personal area networks, IEEE 802.16a WiMax broadband wireless access networks, and more. Therefore, by considering the general framework of TDMA networks our work can be applied without much effort to any TDMA based network, first to offer cooperation, and second to enable a secondary network to share the spectrum owned by the primary network.

\section{Network And Channel Models}

We consider the uplink of a TDMA cellular network as the primary network. The primary network consists of $M_{p}$ source nodes numbered $1,2, \ldots, M_{p}$ communicating with a base station (BS) $d_{p}$ located at the center of the cell as illustrated in Fig. 1. As part of the primary network, $M_{r}$ cognitive relay nodes numbered $1,2, \ldots, M_{r}$ are deployed to help primary nodes forward their packets to the base station. The

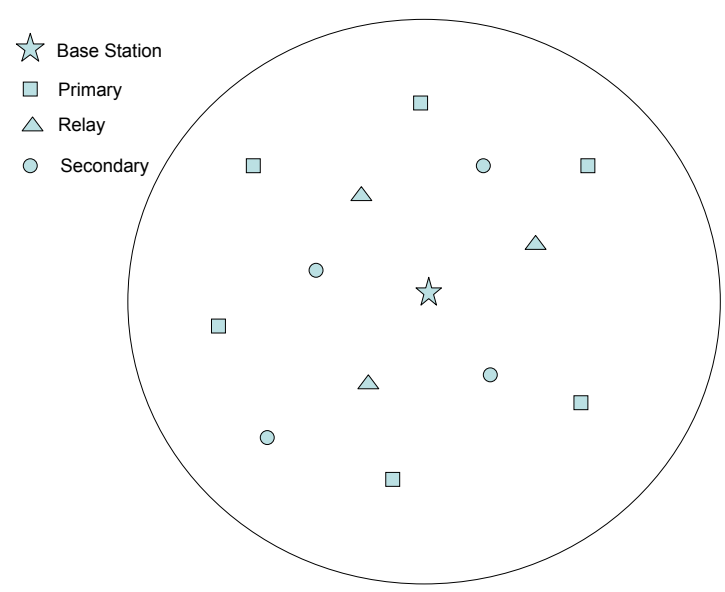

Fig. 1. Network's model

relay nodes will exploit the under-utilized channel resources (time slots in this case) to forward primary packets without incurring any loss in the bandwidth efficiency. A secondary group consisting of $M_{s}$ nodes numbered $1,2, \ldots, M_{s}$ forms an ad-hoc network, and tries to exploit the unutilized channel resources to communicate their own data packets using slotted ALOHA as their MAC protocol. We consider a circular cell of radius $R$. The BS is located at the center of the cell, the different nodes are uniformly distributed within the cell area. Let $\mathcal{M}_{p}=1,2, \ldots, M_{p}$ denote the set of primary nodes, $\mathcal{M}_{r}=1,2, \ldots, M_{r}$ denotes the set of relay nodes, and $\mathcal{M}_{s}=1,2, \ldots, M_{s}$ denotes the set of secondary nodes.

\section{A. Channel Model}

The wireless channel between a node and its destination is modeled as Rayleigh flat fading channel with additive white Gaussian noise. The signal received at a receiving node $j$ from a transmitting node $i$ at time $t$ can be modeled as [3]

$$
y_{i j}^{t}=\sqrt{G_{i} \rho_{i j}^{-\gamma}} h_{i j}^{t} x_{i}^{t}+n_{i j}^{t},
$$

where $G_{i}$ is the transmitting power, assumed to be the same for all nodes, $\rho_{i j}$ denotes the distance between the two nodes, $\gamma$ the path loss exponent, $h_{i j}^{t}$ is the channel fading coefficient between nodes $i$ and $j$ at time $t$ and is modeled as i.i.d zero mean, circularly symmetric complex gaussian random process with unit variance. The term $x_{i}^{t}$ denotes the transmitted packet with average unit power, and $n_{i j}^{t}$ denote i.i.d additive white Gaussian noise processes with zero mean and variance $N_{0}$.

Success and failure of packet reception are characterized by outage events and outage probability. Outage in the link between nodes $i$ and $j$ is defined as the event that the received Signal to Noise ratio (SNR) falls below a certain threshold $\beta$ [10] determined according to the application and the transmitter/receiver structure. If the received SNR is higher than the threshold $\beta$, the receiver is assumed to be able to decode the received message with negligible probability of error. Based on the channel model 1, the outage probability 
can be calculated as follows

$$
\operatorname{Pr}\left\{\mathrm{SNR}_{i j}<\beta\right\}=P_{i j}^{o}=1-\exp \left(-\frac{\beta N_{0}}{G_{i} \rho_{i j}^{\gamma}}\right) .
$$

\section{B. Queuing Model}

Each primary, relay, or secondary node has an infinite buffer for storing fixed length packets. The channel is slotted in time and a slot duration equals the packet transmission time. The arrivals at the $i^{\text {th }}$ primary node's queue $\left(i \in \mathcal{M}_{p}\right)$, and the $j^{\text {th }}$ secondary node's queue $\left(i \in \mathcal{M}_{s}\right)$ are Bernoulli random variables, i.i.d from slot to slot with mean $\lambda_{i}^{p}$ and $\lambda_{j}^{s}$, respectively. Hence, the vector $\Lambda=\left(\lambda_{1}^{p}, \ldots, \lambda_{M_{p}}^{p}, \lambda_{1}^{s}, \ldots, \lambda_{M_{s}}^{s}\right)$ denotes the average arrival rates. Arrival processes are assumed to be independent from one node to another.

Primary users access the channel by dividing the channel resources, time in this case, among them, hence, each node is allocated a fraction of the time. Let $\boldsymbol{\Omega}_{\mathbf{p}}=\left(\omega_{1}^{p}, \omega_{2}^{p}, \ldots, \omega_{M_{p}}^{p}\right)$ denote a resource-sharing vector, where $\omega_{i}^{p} \geq 0$ is the fraction of time allocated to node $i \in \mathcal{M}_{p}$, or it can represent the probability that node $i$ is allocated the whole time slot [11]. The set of all feasible resource-sharing vectors is specified as $\digamma_{p}=\left\{\boldsymbol{\Omega}_{\mathbf{p}}=\left(\omega_{1}^{p}, \omega_{2}^{p}, \ldots, \omega_{M_{p}}^{p}\right) \in \Re^{+M_{p}}: \sum_{i \in \mathcal{M}_{p}} \omega_{i}^{p} \leq 1\right\}$.

In a communication network, the stability of the network's queues is a fundamental performance measure. The system is called stable for a given arrival rate vector and resourcesharing vector pair $(\boldsymbol{\Lambda}, \boldsymbol{\Omega})$ if all the queues are stable, i.e., the primary, secondary nodes and relays' queues are stable. If any queue is unstable, then the whole system is considered unstable. For an irreducible and aperiodic Markov chain with countable number of states, the chain is stable if and only if there is a positive probability for every queue of being empty, i.e., $\lim _{t \rightarrow \infty} \operatorname{Pr}\left\{Q_{i}(t)=0\right\}>0$. (For a rigorous definition of stability under more general scenarios see [12] and [13]). If the arrival and departure processes of a queuing system are strictly stationary, then one can apply Loynes' theorem to check for stability conditions [14]. This theorem states that, if the arrival and departure processes of a queuing system are strictly stationary, and the average arrival rate is less than the average departure rate, then the queue is stable; if the average arrival rate is greater than the average departure rate, then the queue is unstable.

\section{Cognitive Cooperative Protocol with MULTIPLE RELAYS}

In a TDMA system without relays, if a node does not have a packet to transmit, its time slot remains idle, i.e., wasted channel resources. The possibility to utilize these wasted channel resources to provide some sort of spatial diversity and increased reliability to the TDMA system by employing a single cooperative relay node was investigated in [4]. Here we consider the case of a network with multiple relays. We assume that relays can sense the communication channel to detect empty time slots. This assumption is reasonable for the orthogonal multiple-access scheme used, as there is no interference, and the relay can employ coherent or feature detectors that have high detection probability [15]. The second assumption we make is that the errors and delay in packet acknowledgement feedback is negligible, which is reasonable for short length ACK/NACK packets as low rate codes can be employed in the feedback channel.

\section{A. Cooperation Protocol}

First, we describe the relays' cooperation protocol. For the purpose of protocol description and analysis we will assume that the relay selection phase has already taken place, and that every primary node has assigned to it the best relay from the group of available relays. Note that every primary node gets help from only one relay, but a relay might help more than one primary node.

- At the beginning of a time slot, a node transmits the packet at the head of its queue to the destination. Due to the broadcast nature of wireless channel, relays can listen to the transmitted packets by the nodes to the BS.

- If the packet is not received correctly by the BS, a NACK message is fed back from the BS declaring the packet's failure. If the relay assigned to the packet owner was able to decode the packet correctly, it stores the packet in its queue and sends back an ACK message to declare successful reception of the packet at the relay.

- The node drops the packet from its queue if it is correctly received by either the BS or the relay.

- Relays distribute the available time slots in a TDMA fashion. Therefore, if a time slot is detected as empty, this free time slot will be assigned to relay $i \in \mathcal{M}_{r}$ with probability $\omega_{i}^{r}$. As it is the case with TDMA networks, $\boldsymbol{\Omega}_{\mathbf{r}}=$ $\left(\omega_{1}^{r}, \omega_{2}^{r}, \ldots, \omega_{M_{r}}^{r}\right)$ denote a resource-sharing vector, and the set of all feasible resource-sharing vectors is $\digamma_{r}=$ $\left\{\boldsymbol{\Omega}_{\mathbf{r}}=\left(\omega_{1}^{r}, \omega_{2}^{r}, \ldots, \omega_{M_{r}}^{r}\right) \in \Re^{+M_{r}}: \sum_{i \in \mathcal{M}_{r}} \omega_{i}^{r} \leq 1\right\}$.

- Relay $i$ then transmits the packet at the head of its queue.

- We assume that there is enough guard time at the beginning of each time slot that enables sensing, and that channel sensing is error free.

\section{B. Stability Analysis}

In this section we characterize the maximum stable throughput region of the cooperative protocol and compare it against the maximum stable throughput of TDMA without cooperation.

For the whole system to be stable, all queues therein should be stable. Hence, the stability region of the network is the intersection of the stability regions of the source nodes' queues, and the relay nodes' queues.

1) Source Nodes Stability: A source node succeeds in transmitting a packet if either the BS or its assigned relay receive the packet successfully. Therefore, the success probability of node $i$ can be calculated as

$$
\begin{aligned}
P_{i}=\operatorname{Pr}\left\{\overline{O_{i d}} \bigcap \overline{O_{i r_{i}}}\right\}= & \left(1-P_{i d}^{o}\right)+\left(1-P_{i r_{i}}^{o}\right) \\
& -\left(1-P_{i d}^{o}\right)\left(1-P_{i r_{i}}^{o}\right),
\end{aligned}
$$

where $\overline{O_{i j}}$ denotes complement of the event that the channel between node $i$ and receiver $j \in\left(r_{i}, d\right)$ ( $r_{i}$ denotes node $i$ 's relay, and $d$ the $\mathrm{BS}$ ) is in outage (i.e., the event that the 
packet was received successfully). If source node $i$ has no relay assigned to it, its success probability is then given by

$$
P_{i}=\operatorname{Pr}\left\{\overline{O_{i d}}\right\}=1-P_{i d}^{o} .
$$

Since for each queue $i \in \mathcal{M}_{p}$, the queue behaves exactly as in a TDMA system with success probability determined by (3) or (4), and from Loynes's theorem, the primary nodes' stability region $\mathcal{R}_{p}$ is defined as

$$
\begin{array}{r}
\mathcal{R}_{p}=\left\{\left(\lambda_{1}^{p}, \ldots, \lambda_{M_{p}}^{p}\right) \in R^{+M_{p}}: \lambda_{i}^{p}<\omega_{i}^{p} P_{i},\right. \\
\left.\forall i \in \mathcal{M}_{p},\left(\omega_{1}^{p}, \ldots, \omega_{M_{p}}^{p}\right) \in \digamma_{p}\right\},
\end{array}
$$

which can be easily shown to be equivalent to

$$
\mathcal{R}_{p}=\left\{\left(\lambda_{1}^{p}, \ldots, \lambda_{M_{p}}^{p}\right) \in R^{+M_{p}}: \sum_{i \in \mathcal{M}_{p}} \frac{\lambda_{i}^{p}}{P_{i}} \leq 1\right\} .
$$

2) Relay Nodes Stability: In order to apply Loynes' theorem, it is required that the arrival and service processes of the relays' queues are stationary. Let $Q_{j}^{t}$ denote the $j$ th $\left(j \in \mathcal{M}_{r}\right)$ relay queue size at time $t$, then its evolution can be modeled as $Q_{j}^{t+1}=\left(Q_{j}^{t}-Y_{j}^{t}\right)^{+}+X_{j}^{t}$, where $X_{i}^{t}$ represents the number of arrivals in time slot $t$ and $Y_{i}^{t}$ denotes the possibility of serving a packet at this time slot from the $i$ th relay queue $\left(Y_{i}^{t}\right.$ takes values $\left.\{0,1\}\right)$. Function $(\cdot)^{+}$is defined as $(x)^{+}=\max (x, 0)$. Now we establish the stationarity of the arrival and service processes. If source nodes' queues are stable, then by definition the departure processes from these nodes are stationary. A packet departing from a node's queue is stored in the relay's queue (i.e., counted as an arrival) if simultaneously the following two events happen: the nodedestination channel is in outage and the node-relay channel is not in outage. Hence, the arrival process to the queue can be modeled as follows

$$
X_{j}^{t}=\sum_{i \in S_{j}} \mathbf{1}\left[A_{i}^{t} \bigcap\left\{Q_{i}^{t} \neq 0\right\} \bigcap O_{i d} \bigcap \overline{O_{i j}}\right],
$$

where $\mathbf{1}[\cdot]$ is the indicator function, $A_{i}^{t}$ denotes the event that slot $t$ is assigned to source node $i .\left\{Q_{i}^{t} \neq 0\right\}$ denotes the event that node $i$ queue is not empty, i.e., the node has a packet to transmit, and according to Little's theorem [16] it has probability $\lambda_{i}^{p} /\left(\omega_{i}^{p} P_{i}\right)$, where $P_{i}$ is node $i$ success probability and is defined in (3) and (4). Finally, $S_{j}$ denotes the set of source nodes to which relay $j$ assigned to help. The random processes involved in the above expression are all stationary, hence, the arrival process to the relay is stationary. The average arrival rate to the relay's queue can be computed as

$$
\lambda_{j}^{r}=E\left[X_{j}^{t}\right]=\sum_{i \in S_{j}} \lambda_{i}^{p} \frac{P_{i d}^{o}\left(1-P_{i j}^{o}\right)}{P_{i}} .
$$

Similarly, we establish the stationarity of the service process from the $j$ th relay queue. The service process of the relay queue depends by definition on the empty slots available from primary nodes, and the channel from relay to destination being not in outage. By assuming the source nodes' queues to be stable, they offer stationary empty slots (stationary service process) to the relay. Also the channel statistics is stationary, hence, the relay's service process is stationary. The service process of the $j$ th relay's queue can be modeled as

$$
Y_{j}^{t}=\sum_{i \in \mathbf{M}_{p}} \mathbf{1}\left[A_{i}^{t} \bigcap\left\{Q_{i}^{t}=0\right\} \bigcap \overline{O_{j d}^{t}} \bigcap U_{j}^{t}\right],
$$

where $U_{j}^{t}$ is the event that the current idle time slot is assigned to relay $j$ to service its queue, which has probability $\omega_{j}^{r}$ according to the TDMA resource sharing policy employed by the relays. The average service rate of the relay can then be determined from the following equation

$$
\mu_{j}^{r}=E\left[Y_{j}^{t}\right]=\left(1-\sum_{i \in \mathbf{M}_{p}} \frac{\lambda_{i}^{p}}{P_{i}}\right)\left(1-P_{j d}^{o}\right) \omega_{j}^{r} .
$$

Using Loynes' theorem, the stability condition for the $j$ th relay queue is $\lambda_{j}^{r}<\mu_{j}^{r}$. The stability region $\mathcal{R}_{r}$ of the system comprised of the relays' queues is then defined as

$$
\begin{array}{r}
\mathcal{R}_{r}=\left\{\left(\lambda_{1}^{p}, \ldots, \lambda_{M_{p}}^{p}\right) \in R^{+M_{p}}: \lambda_{j}^{r}<\mu_{j}^{r},\right. \\
\left.\forall j \in \mathcal{M}_{r},\left(\omega_{1}^{r}, \ldots, \omega_{M_{r}}^{r}\right) \in \digamma_{r}\right\},
\end{array}
$$

which can be easily shown to be equivalent to

$$
\begin{aligned}
\mathcal{R}_{r}= & \left\{\left(\lambda_{1}^{p}, \ldots, \lambda_{M_{p}}^{p}\right) \in R^{+M_{p}}:\right. \\
& \left.\sum_{j \in \mathcal{M}_{r}} \frac{\sum_{i \in S_{j}} \lambda_{i}^{p} \frac{P_{i d}^{o}\left(1-P_{i j}^{o}\right)}{P_{i}}}{\left(1-\sum_{i \in \mathcal{M}_{p}} \frac{\lambda_{i}^{p}}{P_{i}}\right)\left(1-P_{j d}^{o}\right)} \leq 1\right\} .
\end{aligned}
$$

Finally, the maximum stable throughput region of the complete system defined by the source nodes and relays queues is given by the intersection of the maximum stable throughput regions of source nodes queues and relays queues, which can be shown to be equal to $\mathcal{R}=\mathcal{R}_{p} \bigcap \mathcal{R}_{r}=\mathcal{R}_{r}$.

From (12) it is noted that the stability region for the cooperative protocol is bounded by a hyperplane. Since the stability of TDMA is also determined by a hyperplane, when comparing both stability regions it is enough to compare the intersection of these hyperplanes with the coordinate axes . Considering the $i^{t h}$ source node, this intersection for the cooperative protocol is equal to

$$
\lambda_{i}^{p *}(\mathrm{Coop})=\frac{P_{i}\left(1-P_{j d}^{o}\right)}{P_{i d}^{o}\left(1-P_{i j}^{o}\right)+\left(1-P_{j d}^{o}\right)},
$$

where it was assumed that relay node $j$ is assigned to source node $i$. The corresponding value for TDMA is given by

$$
\lambda_{i}^{p *}(\mathrm{TDMA})=1-P_{i d}^{o} .
$$

It is clear that the stability region for TDMA is completely contained inside the stability region of the cooperative protocol if $\lambda_{i}^{p *}$ (Coop) $>\lambda_{i}^{p *}$ (TDMA) for all $i \in \mathcal{M}_{p}$. Using (13) and (14), this condition is equivalent to

$$
P_{j d}^{o}<P_{i d}^{o}
$$

These conditions have the following intuitive explanation. If the channel between the relay and destination has higher success probability that the channel between the terminal and destination, then it is better to have the relay help the terminal transmit its packets. Note that (15) implies that TDMA can offer better performance for the node whose outage probability does not satisfy (15). 


\section{Relay Selection}

The nearest neighbor is one of the widely considered relay selection criterion in cooperative communications [17]. Applying the nearest neighbor relay selection criterion to our network model results in the maximization of the source node service rate without taking relays' queues into consideration. In order to maximize the whole network's stability region, the relay selection process should be able to take the service rates of the relays into consideration. Intuitively, it is beneficial (from a stability point of view) to favor the relays with higher service rates over the ones with lower service rates. To take the relay-destination link into consideration, we propose the following criterion where source node $i$ selects a relay according to

$$
\begin{array}{r}
\arg \max _{j \in \mathcal{M}_{r}}\left(1-P_{i j}^{o}\right)\left(1-P_{j d}^{o}\right) \\
\text { s.t. } P_{j d}^{o}<P_{i d}^{o},
\end{array}
$$

i.e., node $i$ selects the relay that maximizes the overall packet success probability over both source-relay and relay-destination links, under the constraint that the relaydestination link has a higher success probability that the source-destination link. Using the definition of the outage probability, it can be shown that the relay selection criterion of (16) is equivalent to

$$
\begin{array}{r}
\arg \min _{j \in \mathcal{M}_{r}} \rho_{i j}+\rho_{j d} \\
\text { s.t. } \rho_{j d}<\rho_{i d},
\end{array}
$$

where $\rho_{i j}$ is the distance between source node $i$ and relay node $j, \rho_{i d}$ the distance between source node $i$ and the destination, and $\rho_{j d}$ the distance between relay node $j$ and the destination. Therefore, the maximum success probability criterion reduces to a minimization of the sum of the source-relay and relaydestination distances.

\section{Opportunistic Multiple Access for Secondary NODES}

In the previous section, the problem of utilizing the idle channel resources to enable cognitive relays to help source nodes forward their packets was considered. Aside from being used by relays, these idle channel resources could be used by a group of secondary (unlicensed) nodes to transmit their own data packets. Therefore, the use if these idle channel resources (time slots, in our network) offers either diversity to the primary nodes through the group of relays, or multiplexing through the group of secondary nodes that send new information over the channel.

In this section, we study the effect of sharing the idle time slots between relays and secondary nodes on the performance of both primary and secondary networks. Mainly, how the secondary network's throughput is affected when part of the idle channel resources are used by the relays. And, how the primary network throughput is affected when secondary transmissions interfere with relay transmissions. Furthermore, we study the possibility that secondary nodes work as relays for the primary network. By working as relays, the secondary nodes aim at creating more transmission opportunities for

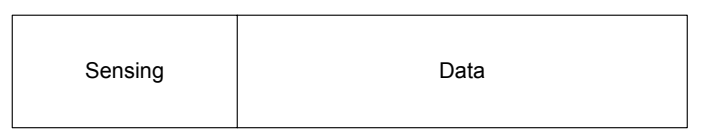

Fig. 2. Time slot structure, showing the sensing period used by the relays to detect primary presence.

themselves by helping primary nodes empty their queues at a faster rate.

The secondary network consists of $M_{s}$ nodes forming an ad-hoc network, in which nodes are grouped into sourcedestination pairs where each source node communicates with its associated destination node. To access the channel, secondary nodes will use the beacon sent by primary nodes at the beginning of each time slot, as shown in Fig. 2, to detect primary activity. As with the relays, we assume that the primary detection process is error free. to share the idle time slots among the secondary network, secondary nodes employ slotted ALOHA as a MAC protocol. Therefore, whenever an idle slot is detected, secondary nodes with nonempty queues will attempt to transmit their packets with channel access probability $\alpha_{s}$.

Since both relay and secondary nodes sense the channel at the beginning of each time slot, it is not necessary that secondary nodes will be able to detect relay transmissions. In such situations, secondary packets will collide with relay packets. To take these collision events into consideration, we will study two extreme cases. The first is when the secondary nodes are always unable to detect relays transmissions, thus always, colliding with relays if they decide to transmit at the same time slot. The second case occurs when secondary nodes are all the time able to detect relays presence successfully, thus no interference at all. The study of these two cases enables us to find inner and outer bounds on the maximum stable throughput region of the network.

Furthermore, we consider the tradeoff between the amount of help offered to the primary network through relays, and the achievable throughput of the secondary network. To study this tradeoff, we consider the case where relays limit their access to the channel, therefore, providing secondary nodes with uncontested access to the idle time slots. This is made possible by letting relays make their transmission attempts in an empty time slot with probability an access $\alpha_{r}$. In other words, when a relay has a packet to transit, and it encounters an idle time slot, it will transmit its packet with probability $\alpha_{r}$, and defer transmitting, in order to offer allow secondary nodes to use that slot, with probability $1-\alpha_{r}$.

\section{A. Case I: No Interference}

Here we consider the case when secondary nodes are always able to successfully detect relays transmissions. Therefore, no interference is exhibited by relay nodes from secondary transmissions. This can be seen as a best case scenario and helps in characterizing an upper bound on the performance of both primary and secondary networks.

In order to share resources with secondary nodes, and enable secondary nodes to access the idle time slots, relays will limit their access to the channel by utilizing a transmission 
probability $\alpha_{r}$. In other words, when a relay detects an empty time slot, it transmits the packet at the head of its queue with probability $\alpha_{r}$, and remains silent with probability $1-\alpha_{r}$. In this case, TDMA is still used to organize relays access to idle time slots, and we assume that all relays will use the same probability $\alpha_{r}$. Therefore, relays will collectively use a fraction $\alpha_{r}$ of the idle time slots to offer help to primary nodes, and secondary nodes will have a guaranteed access to at least a fraction $1-\alpha_{r}$ of the idle time slots. The actual figure will be higher since relays will not have packets to transmit all the time. It should also be noted that, since all relays have the same access probability, this scheme will not affect the relay selection process.

1) Primary Network Stability Analysis: Since service processes of the primary nodes are not affected by how relays access the channel, the stability region of the system comprised of primary queues is defined as in (6).

To characterize the stability region of the system composed of relays queues, we first note that the arrival process to a relay queue is not affected by the relay's channel access mechanism. Therefore, the arrival process for relay queue $j \in \mathcal{M}_{r}$ is defined as in (7), and its average arrival rate given by (8). For the $j^{\text {th }}$ relay service process, stationarity of the service process could easily be established using the same arguments used in the previous section. Therefore, the service process of the $j^{\text {th }}$ relay's queue can be modeled as

$$
Y_{j}^{t}=\sum_{i \in \mathcal{M}_{p}} \mathbf{1}\left[A_{i}^{t} \bigcap\left\{Q_{i}^{t}=0\right\} \bigcap \overline{O_{j d}^{t}} \bigcap U_{j}^{t} \bigcap P_{r}\right],
$$

where $U_{j}^{t}$ is the event that the current idle time slot is assigned to relay $j$ to service its queue, which has probability $\omega_{j}^{r}$ according to the TDMA resource sharing policy employed by the relays, and $P_{r}$ is the event that relay $j$ has permission to access the channel in the current time slot, which has a probability $\alpha_{r}$. The average service rate of the relay can then be determined from the following equation

$$
\mu_{j}^{r}=E\left[Y_{j}^{t}\right]=\left(1-\sum_{i \in \mathcal{M}_{p}} \frac{\lambda_{i}^{p}}{P_{i}}\right)\left(1-P_{j d}^{o}\right) \omega_{j}^{r} \alpha_{r} .
$$

Using Loynes' theorem, the stability condition for the $j^{\text {th }}$ relay queue is $\lambda_{j}^{r}<\mu_{j}^{r}$, and since the set of relay nodes form a TDMA system, the stability region $\mathcal{R}_{r}$ of the system comprised of the relays' queues can be shown to be defined as follows,

$$
\begin{aligned}
\mathcal{R}_{r}= & \left\{\left(\lambda_{1}^{p}, \ldots, \lambda_{M_{p}}^{p}\right) \in R^{+M_{p}}:\right. \\
& \left.\sum_{j \in \mathcal{M}_{r}} \frac{\sum_{i \in S_{j}} \lambda_{i}^{p} \frac{P_{i d}^{o}\left(1-P_{i j}^{o}\right)}{P_{i}}}{\left(1-\sum_{i \in \mathcal{M}_{p}} \frac{\lambda_{i}^{p}}{P_{i}}\right)\left(1-P_{j d}^{o}\right) \alpha_{r}} \leq 1\right\},
\end{aligned}
$$

which can also be shown to be equal to the stability region of the whole primary network (primary and relay nodes).

From (20) it is noted that the stability region in this case is also bounded by a hyperplane. The intersection with the $i^{\text {th }}$ coordinate axis gives the maximum allowable arrival rate for the $i^{\text {th }}$ source node, which is equal to

$$
\lambda_{i}^{p *}(\mathrm{Coop})=\frac{P_{i}\left(1-P_{j d}^{o}\right) \alpha_{r}}{P_{i d}^{o}\left(1-P_{i j}^{o}\right)+\left(1-P_{j d}^{o}\right) \alpha_{r}}
$$

which is a monotonically increasing function in $\alpha_{r}$. Therefore, from the point of view of primary network stability, it is always beneficial to assign most of the idle resources to relays.

The condition in (15), defining when the cooperative protocol outperforms TDMA, translates into

$$
\left(1-P_{j d}^{o}\right) \alpha_{r}>\left(1-P_{i d}^{o}\right),
$$

which tells us that, by limiting their access to the channel, relays appear to primary nodes as having higher outage probabilities. Therefore, according to the value of $\alpha_{r}$ and different relays' outage probabilities, some of the relays might be rendered unusable, and situations might arise in which no relay is used at all.

2) Secondary Network Stability Analysis: Switching to the analysis of the secondary nodes stability, we recall that the secondary network consists of $M_{s}$ nodes numbered $1,2, \ldots, M_{s}$, and having average arrival rates $\left[\lambda_{1}^{s}, \ldots, \lambda_{M_{s}}^{s}\right]$. Upon the detection of an idle time slot, a node with a non-empty queue will try to transmit the packet at the head of its queue with access probability $\alpha_{s}$. A node's sensing and channel access decisions are independent from other nodes. We further assume for mathematical tractability that all nodes have the same access probability $\alpha_{s}$.

To study the stability region of secondary nodes, we note first that, since secondary nodes are employing slotted ALOHA for multiple access, the secondary nodes queues are interacting. In other words, the service rate of a given queue is dependent on the state of all other queues, i.e., whether they are empty or not. Studying the stability conditions for interacting queues is a difficult problem that has been addressed for ALOHA systems [13], [18]. The concept of dominant systems was introduced and employed in [13] to help find bounds on the stability region of ALOHA with collision channel. The dominant system in [13] was defined by allowing a set of terminals with no packets to transmit to continue transmitting dummy packets. In this manner, the queues in the dominant system stochastically dominate the queues in the original system. Or in other words, with the same initial conditions for queue sizes in both the original and dominant systems, the queue sizes in the dominant system are not smaller than those in the original system.

To study the stability of the interacting system of queues consisting of secondary nodes queues, we make use of the dominant system approach to decouple the interaction between queues. We define the dominant system as follows

- Arrivals at each queue in the dominant system are the same as in the original system.

- Time slots assigned to primary node $i \in \mathcal{M}_{p}$ are identical in both systems.

- The outcomes of the "coin tossing" (that determines transmission attempts of relay and secondary nodes) in every slot are the same.

- Channel realizations for both systems are identical.

- The noise generated at the receiving ends of both systems is identical. 
- In the dominant system, secondary nodes attempt to transmit dummy packets when their queues are empty.

For a proof that the dominant system's stability conditions are necessary and sufficient for the stability of the original system see the Appendix.

The service process of a secondary node depends on the idle time slots unused by the primary and relay nodes. Therefore, the service process of the $k^{\text {th }}$ secondary node can be modeled as

$$
\begin{aligned}
& Y_{k}^{t}=\sum_{i \in \mathcal{M}_{p}} \sum_{j \in \mathcal{M}_{r}} \mathbf{1}\left[A_{i}^{t} \bigcap\left\{Q_{i}^{t}=0\right\} \bigcap U_{j}^{t}\right. \\
&\left.\bigcap\left\{\overline{\left\{Q_{j}^{t} \neq 0\right\} \bigcap P_{r}}\right\} \bigcap \overline{O_{k d}^{t}} \bigcap P_{s} \bigcap_{l \in \mathcal{M}_{s} \backslash k}\left\{\overline{P_{s}}\right\}\right],
\end{aligned}
$$

which is the event that the primary node for which the current time slot is assigned has an empty queue, and the relay for which the current time slot has either an empty queue or does not have permission to transmit (the event $U_{j}^{t} \cap\left\{\overline{\left\{Q_{j}^{t} \neq 0\right\} \cap P_{r}}\right\}$. Event $P_{s}$ is the event that a secondary node has a permission to transmit, which has a probability $\alpha_{s}$. Therefore, the event $\bigcap P_{s} \bigcap_{l \in \mathcal{M}_{s} \backslash k}\left\{\overline{P_{s}}\right\}$ is that only one secondary node is transmitting in the current time slot; otherwise a collision will occur and all packets involved will be lost. Finally, $\overline{O_{k d}^{t}}$ denotes the event that the $k^{\text {th }}$ secondary node link to its destination is not in outage.

Assuming that primary and relay nodes' queues are stable, then they offer stationary empty slots. Also the channel statistics are stationary; hence, the secondary service process is stationary. The average secondary service rate is then given by

$$
\begin{aligned}
\mu_{k}^{s}=\left(1-\sum_{i \in \mathcal{M}_{p}} \frac{\lambda_{i}^{p}}{P_{i}}-\right. & \left.\sum_{j \in \mathcal{M}_{r}} \frac{\sum_{i \in S_{j}} \lambda_{i}^{p} \frac{P_{i d}^{o}\left(1-P_{i j}^{o}\right)}{P_{i}}}{\left(1-P_{j d}^{o}\right)}\right) \\
& \times \alpha_{s}\left(1-\alpha_{s}\right)^{M_{s}-1}\left(1-P_{k d}^{o}\right) .
\end{aligned}
$$

From (24), it can be easily shown that the optimum value for the secondary access probability is $\alpha_{s}=1 / M_{s}$.

Using Loyne's theorem along with (24), and from (20), the stability region of the system defined by the primary nodes, relay nodes, and secondary nodes can be written as

$$
\begin{aligned}
\mathcal{R}= & \mathcal{R}_{r} \bigcap \mathcal{R}_{s}=\left\{\left(\lambda_{1}^{p}, \ldots, \lambda_{M_{p}}^{p}, \lambda_{1}^{s}, \ldots, \lambda_{M_{s}}^{s}\right) \in R^{+\left(M_{p}+M_{s}\right)}:\right. \\
& \sum_{j \in \mathcal{M}_{r}} \frac{\sum_{i \in S_{j}} \lambda_{i}^{p} \frac{P_{i d}^{o}\left(1-P_{i j}^{o}\right)}{P_{i}}}{\left(1-\sum_{i \in \mathcal{M}_{p}} \frac{\lambda_{i}^{p}}{P_{i}}\right)\left(1-P_{j d}^{o}\right)} \alpha_{r} \leq 1, \\
& \lambda_{k}^{s} \leq\left(1-\sum_{i \in \mathcal{M}_{p}} \frac{\lambda_{i}^{p}}{P_{i}}-\sum_{j \in \mathcal{M}_{r}} \frac{\sum_{i \in S_{j}} \lambda_{i}^{p} \frac{P_{i d}^{o}\left(1-P_{i j}^{o}\right)}{P_{i}}}{\left(1-P_{j d}^{o}\right)}\right) \\
& \left.\times \frac{1}{M_{s}}\left(1-\frac{1}{M_{s}}\right)^{M_{s}-1}\left(1-P_{k d}^{o}\right), k \in \mathcal{M}_{s}\right\} .
\end{aligned}
$$

Dependence of secondary nodes service rates in (24) on the parameter $\alpha_{r}$ appears only through primary nodes success probabilities $P_{i}$ defined in (3) and (4), and relay nodes arrival rates, which are dependent on $\alpha_{r}$ through the relay assignment process. Clearly, a higher $\alpha_{r}$ will result in primary nodes getting better service from relays; thus, primary queues will have higher services rates. Therefore, there will be a higher probability that primary queues are empty. Since relay service rates explicitly depend on $\alpha_{r}$ as shown in (19), higher $\alpha_{r}$ will also mean a higher probability of empty relay queues. This results in more idle time slots for secondary nodes to exploit.

3) Case II: Maximum Interference: Here we consider the worst case scenario where secondary nodes cannot sense relay transmissions at all. In this case, collisions between relays and secondary transmissions are inevitable. In case of a collision all packets involved are lost, and a retransmission is necessary. This scenario helps us characterize a lower bound on the performance of both primary and secondary networks.

Again, to study the tradeoff between assigning idle resources to relays or secondary nodes, the case where relays limit their access to the idle time slot and have access probability $\alpha_{r}$ is considered. Since the cognitive principle is based on the idea that the presence of the secondary system should be transparent to the primary system, the secondary nodes access probability $\alpha_{s}$ will now play a crucial role of limiting secondary interference to the primary network.

Because of the possible collisions between secondary and relay transmissions, relay and secondary nodes queues form a system of interacting queues.

To study the stability of the interacting system of queues consisting of the relay and secondary nodes queues, we make use of the dominant system approach to decouple the interaction between the queues. Because relay nodes' transmissions can cause collisions with secondary transmissions, the dominant system in this case will be similar to the one used in the previous section except that we define two dominant systems $D_{1}$ and $D_{2}$,

- In $D_{1}$ relays will attempt to transmit dummy packets if their queues are empty. Since secondary queues are interacting among themselves (an interaction that needs to be decoupled as well), secondary nodes will attempt to transmit dummy packets only if they are informed (with the aid of a "genie") that relays are not transmitting in the current time slot.

- In $D_{2}$, secondary nodes attempt to transmit dummy packets when their queues are empty, and relays operate normally.

Stability conditions for the above defined dominant system could be shown to be necessary and sufficient for the stability of the original system through similar arguments to the ones presented in the Appendix.

4) Dominant System $D_{1}$ : Under this dominant system, relays will be transmitting dummy packets if their queues are empty. Since service processes of the primary nodes are not affected by how relays access the channel, the stability region of the system comprised of primary queues is defined as in (6).

We start by characterizing the stability region of the system defined by the relays' queues. As in the previous section, the average arrival rate to the relay is unchanged and is given by (8). Relays' service processes now depend on the state of secondary queues in addition to the empty slots available from 
primary nodes, and the channel from a relay to the destination not being in outage. The service process of the $j^{\text {th }}$ relay queue can then be modeled as

$$
\begin{array}{r}
Y_{j}^{t}=\sum_{i \in \mathcal{M}_{p}} \mathbf{1}\left[A_{i}^{t} \bigcap\left\{Q_{i}^{t}=0\right\} \bigcap U_{j}^{t} \bigcap P_{r} \bigcap \overline{O_{j d}^{t}}\right. \\
\bigcap_{k \in \mathcal{M}_{s}}\left\{\overline{\left.\left.\left\{Q_{k}^{t} \neq 0\right\} \bigcap P_{s}\right\}\right],}\right.
\end{array}
$$

which accounts for the events that, the primary node owning the current time slot has an empty queue, the current time slot is assigned to relay $j$, the relay has permission to transmit, the relay-destination link is not in outage, and finally, no secondary node is transmitting, which is either due to empty queues or lack of permission to transmit. The average service rate of the $j^{\text {th }}$ relay is then given by

$$
\begin{array}{r}
\mu_{j}^{r}=E\left[Y_{j}^{t}\right]=\left(1-\sum_{i \in \mathcal{M}_{p}} \frac{\lambda_{i}}{P_{i}}\right) \omega_{j}^{r} \alpha_{r}\left(1-P_{j d}^{o}\right) \\
\prod_{k \in \mathcal{M}_{s}}\left(1-\frac{\lambda_{k}^{s}}{\mu_{k}^{s}} \alpha_{s}\right) .
\end{array}
$$

Next, we consider the service processes for the secondary queues. Beside the idle time slots unused by the primary nodes and other secondary nodes queues, the service process of a secondary node now depends on whether or not relays have permission to transmit. Therefore, the service process of the $k^{t h}$ secondary node can be modeled as

$$
\begin{gathered}
Y_{k}^{t}=\sum_{i \in \mathcal{M}_{p}} \sum_{j \in \mathcal{M}_{r}} \mathbf{1}\left[A_{i}^{t} \bigcap\left\{Q_{i}^{t}=0\right\} \bigcap U_{j}^{t} \bigcap \overline{P_{r}} \bigcap \overline{O_{k d}^{t}}\right. \\
\left.\bigcap P_{s} \bigcap_{l \in \mathcal{M}_{s} \backslash k}\left\{\overline{P_{s}}\right\}\right]
\end{gathered}
$$

which is the event that the primary node for which the current time slot is assigned has an empty queue, the relay has no permission to transmit, the $k^{\text {th }}$ secondary node has permission to transmit, all other secondary nodes do not have permission, and the secondary-destination link is not in outage. The average secondary service rate is then given by

$$
\begin{aligned}
& \mu_{k}^{s}=E\left[Y_{k}^{t}\right]=\left(1-\sum_{i \in \mathcal{M}_{p}} \frac{\lambda_{i}^{p}}{P_{i}}\right)\left(1-\alpha_{r}\right) \alpha_{s}\left(1-\alpha_{s}\right)^{M_{s}-1} \\
& \times\left(1-P_{k d}^{o}\right) .
\end{aligned}
$$

Using Loynes' theorem and (8), (27), and (29), the stability region for the dominant system $D_{1}$ for a given $\alpha_{r}$ and $\alpha_{s}$ is given by Equation (30) (shown on top of next page).

5) Dominant System $D_{2}$ : Under this dominant system, secondary nodes will be transmitting dummy packets if their queues are empty. As it is the case in previous sections, the stability region of the system comprised of primary queues is defined as in (6).

To characterize the stability region of the system defined by the relays queues, we note that as in the previous section, the average arrival rate to the relay is unchanged and is given by (8). The service process of the $j^{\text {th }}$ relay queue is modeled as

$$
\begin{array}{r}
Y_{j}^{t}=\sum_{i \in \mathcal{M}_{p}} \mathbf{1}\left[A_{i}^{t} \bigcap\left\{Q_{i}^{t}=0\right\} \bigcap U_{j}^{t} \bigcap P_{r} \bigcap \overline{O_{j d}^{t}}\right. \\
\left.\bigcap_{k \in \mathcal{M}_{s}}\left\{\overline{\bigcap P_{s}}\right\}\right],
\end{array}
$$

which differs from (26) in the term accounting for the state of secondary queues. Here we have only the event that no secondary node has permission to transmit, because even if the queues are empty, secondary nodes continue to transmit dummy packets. The average service rate of the $j^{t h}$ relay is then given by

$\mu_{j}^{r}=E\left[Y_{j}^{t}\right]=\left(1-\sum_{i \in \mathcal{M}_{p}} \frac{\lambda_{i}}{P_{i}}\right) \omega_{j}^{r} \alpha_{r}\left(1-P_{j d}^{o}\right)\left(1-\alpha_{s}\right)^{M_{s}}$.

Next, we consider the service processes for the secondary queues. Here the secondary service process is dependent on the states of different relay queues. Therefore, the service process of the $k^{t h}$ secondary node can be modeled as

$$
\begin{aligned}
Y_{k}^{t}= & \sum_{i \in \mathcal{M}_{p}} \sum_{j \in \mathcal{M}_{r}} \mathbf{1}\left[A_{i}^{t} \bigcap\left\{Q_{i}^{t}=0\right\} \bigcap U_{j}^{t}\right. \\
& \left.\bigcap \overline{\left\{Q_{j}^{t} \neq 0\right\} \bigcap P_{r}} \bigcap \overline{O_{k d}^{t}} \bigcap P_{s} \bigcap_{l \in \mathcal{M}_{s} \backslash k}\left\{\overline{P_{s}}\right\}\right],
\end{aligned}
$$

and the average secondary service rate is then given by

$$
\begin{array}{r}
\mu_{k}^{s}=\left(1-\sum_{i \in \mathcal{M}_{p}} \frac{\lambda_{i}^{p}}{P_{i}}-\sum_{\substack{j \in \mathcal{M}_{r}\\
}} \frac{\sum_{i \in S_{j}} \lambda_{i}^{p} \frac{P_{i d}^{o}\left(1-P_{i j}^{o}\right)}{P_{i}}}{\left(1-P_{j d}^{o}\right)\left(1-\alpha_{s}\right)^{M_{s}}}\right) \\
\times \alpha_{s}\left(1-\alpha_{s}\right)^{M_{s}-1}\left(1-P_{k d}^{o}\right) .
\end{array}
$$

Using Loynes' theorem and (8), (32), and (34), the stability region for the dominant system $D_{2}$ for a given $\alpha_{r}$ and $\alpha_{s}$ can be written as follows,

$$
\begin{aligned}
& \mathcal{R}\left(D_{2}\right)=\left\{\left(\lambda_{1}^{p}, \ldots, \lambda_{M_{p}}^{p}, \lambda_{1}^{s}, \ldots, \lambda_{M_{s}}^{s}\right) \in R^{+\left(M_{p}+M_{s}\right)}:\right. \\
& \sum_{j \in \mathcal{M}_{r}} \frac{\sum_{i \in S_{j}} \lambda_{i}^{p} \frac{P_{i d}^{o}\left(1-P_{i j}^{o}\right)}{P_{i}}}{\left(1-\sum_{i \in \mathcal{M}_{p}} \frac{\lambda_{i}^{p}}{P_{i}}\right) \alpha_{r}\left(1-P_{j d}^{o}\right)\left(1-\alpha_{s}\right)^{M_{s}}} \leq 1, \\
& \lambda_{k}^{s} \leq\left(1-\sum_{i \in \mathcal{M}_{p}} \frac{\lambda_{i}^{p}}{P_{i}}-\sum_{j \in \mathcal{M}_{r}} \frac{\sum_{i \in S_{j}} \lambda_{i}^{p} \frac{P_{i d}^{o}\left(1-P_{i j}^{o}\right)}{P_{i}}}{\left(1-P_{j d}^{o}\right)\left(1-\alpha_{s}\right)^{M_{s}}}\right) \\
& \left.\times \alpha_{s}\left(1-\alpha_{s}\right)^{M_{s}-1}\left(1-P_{k d}^{o}\right), k \in \mathcal{M}_{s}\right\} .
\end{aligned}
$$

Finally, the whole stability region can be determined by taking the union over all possible values of $\alpha_{s}$ as follows,

$$
\mathcal{R}=\bigcup_{\alpha_{s} \in[0,1]}\left\{\mathcal{R}\left(D_{1}\right) \bigcup \mathcal{R}\left(D_{2}\right)\right\} .
$$

The dependence of the stability region of (36) on the resource assignment parameter $\alpha_{r}$ is very complex to characterize. Looking at the stability conditions of the system $D_{1}$, 


$$
\begin{aligned}
& \mathcal{R}\left(D_{1}\right)=\left\{\left(\lambda_{1}^{p}, \ldots, \lambda_{M_{p}}^{p}, \lambda_{1}^{s}, \ldots, \lambda_{M_{s}}^{s}\right) \in R^{+\left(M_{p}+M_{s}\right)}\right.: \sum_{j \in \mathcal{M}_{r}} \frac{\sum_{i \in S_{j}} \lambda_{i}^{p} \frac{P_{i d}^{o}\left(1-P_{i j}^{o}\right)}{P_{i}}}{\left(1-\sum_{i \in \mathcal{M}_{p}} \frac{\lambda_{i}^{p}}{P_{i}}\right)\left(1-P_{j d}^{o}\right) \alpha_{r} \prod_{k \in \mathcal{M}_{s}}\left(1-\frac{\lambda_{k}^{s}}{\mu_{k}^{s}} \alpha_{s}\right)} \leq 1, \\
&\left.\lambda_{k}^{s} \leq\left(1-\sum_{i \in \mathcal{M}_{p}} \frac{\lambda_{i}^{p}}{P_{i}}\right)\left(1-\alpha_{r}\right) \alpha_{s}\left(1-\alpha_{s}\right)^{M_{s}-1}\left(1-P_{k d}^{o}\right), k \in \mathcal{M}_{s}\right\}
\end{aligned}
$$

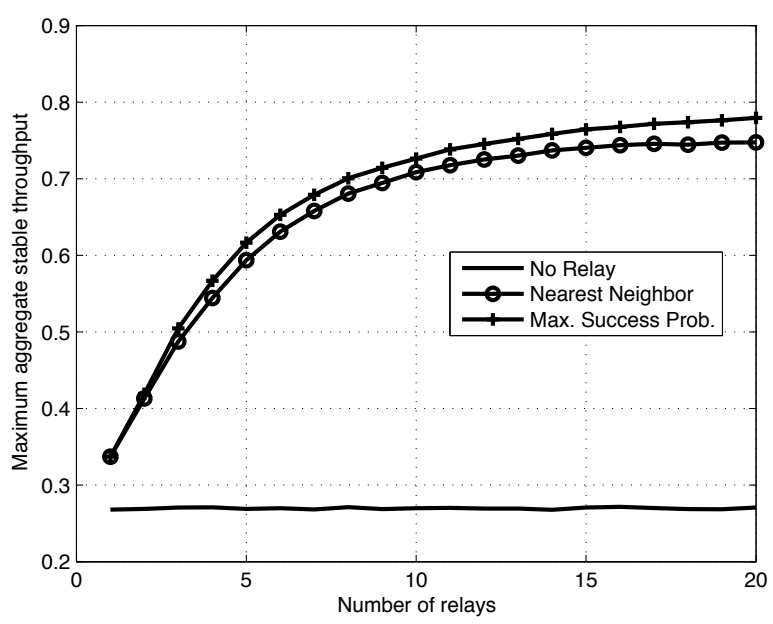

Fig. 3. Maximum aggregate stable throughput vs. number of relays. $M_{p}=$ 20 primary nodes.

it can be noted from (29) that, on one hand, the dependence of the secondary queues service rates on the primary queues service rates makes it beneficial to assign more idle slots to relays. On the other hand, because of the possible collisions with relay transmissions, which is modeled with the term $\left(1-\alpha_{r}\right)$ in $(29)$, it is better from a secondary network point of view to reduce the amount of resources assigned to relays. Similarly, from (27) it is noted that the relay's service rate has two competing components that depend on $\alpha_{r}$. The first rises from primary queues service rates which are increasing in $\alpha_{r}$. The second is the probability that secondary queues are empty, in which as discussed above, its dependence on $\alpha_{r}$ is not easily identified. If we then look at the dominant system $D_{2}$, it will be immediately clear from (32) and (34) that both primary and secondary nodes will benefit from assigning more resources to relays.

\section{RESUlts AND Discussions}

First we present results for the proposed selection schemes by considering the following scenario. $M_{p}=20$ source nodes, and $M_{r}=1, \ldots, 20$ relay nodes are deployed uniformly in a circular cell of radius $R=200 \mathrm{~m}$, with the BS located at the center of the cell. The propagation path loss is taken equal to $\gamma=3.7$ and the SNR threshold $\beta=35 \mathrm{~dB}$. The transmitted signal power is $G=100 \mathrm{~mW}$, and the noise power is $N_{0}=10^{-11}$. For ease of illustration we consider the aggregate network arrival rate $\lambda_{p}=\sum_{i} \lambda_{p}^{i}, i \in \mathcal{M}_{p}$.

Fig. 3 compares the maximum stable throughput of the cooperative versus non-cooperative networks as a function of

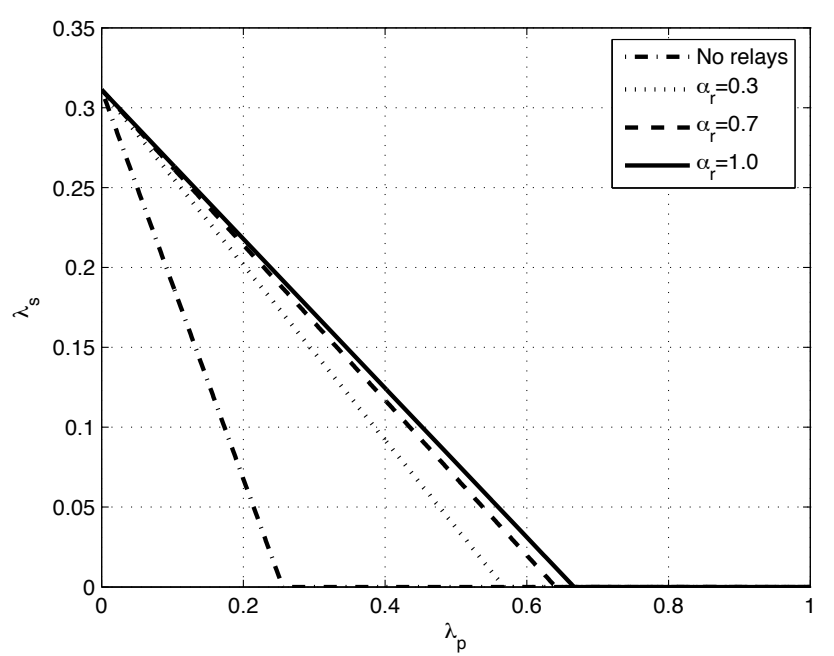

Fig. 4. System stability region with and without cooperation for different values of $\alpha_{r}$.

the number of relays in the network. Furthermore, it compares the performance of the two proposed relay selection schemes. It is clear that the cooperative protocol outperforms its noncooperative counterpart; even with a single relay (which of course is not helping all the nodes) a $25 \%$ increase in throughput is achieved. As the number of relays increases we notice a fast increase in throughput; for example, with 5 relays the throughput is increased by $128 \%$. Increasing the number of relays to 10 leads to a $167 \%$ increase in throughput. This is mainly because increasing the number of relays increases the number of source nodes that are getting help from these relays, hence leading to higher throughput.

From Fig. 3, it can be seen that the "maximum success probability" relay selection criterion outperforms the"nearest neighbor" criterion by a margin of $3 \%$ to $4 \%$ on average. Furthermore, it is noted that the gap between the two criteria increases with increasing number of relays. This is due to the fact that with an increased relay density in the network, there will be a higher probability that a source node finds a relay at or near the optimal relay position corresponding to that source node. While the "maximum success probability" criterion will be able to select the relay at the optimal (or near optimal) location, the "nearest neighbor criterion" will always pick the closest relay to the source node.

Next we consider the network stability region under the ideal case of no collisions between secondary and relay nodes. In order to be able to visualize the network's stability region, which has in general $M_{p}+M_{s}$ dimensions, we plot the maximum aggregate primary arrival rate $\lambda_{p}=\sum_{i} \lambda_{i}^{p}$, for 


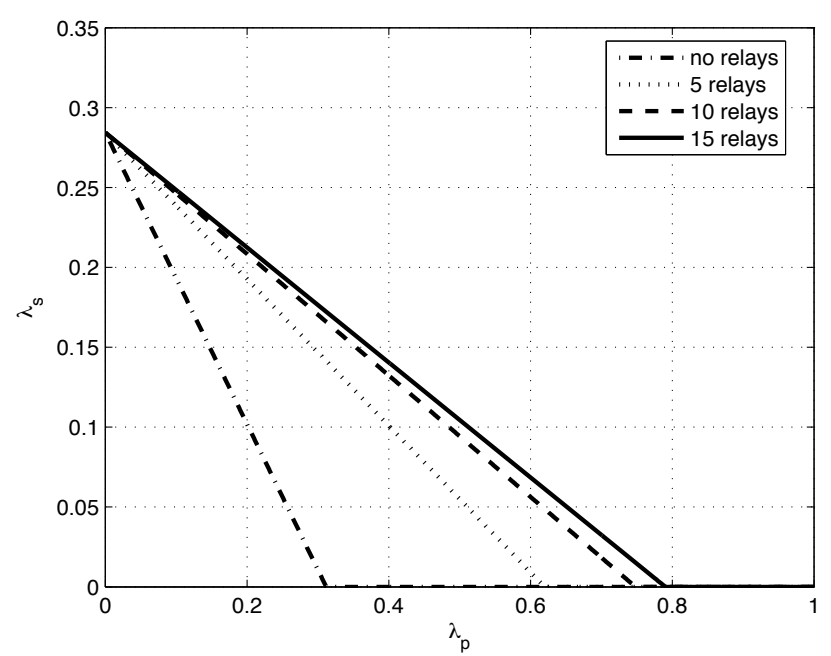

Fig. 5. Stability region with different number of relays. $M_{p}=20$ primary nodes and $M_{s}=10$ secondary nodes.

$i \in \mathcal{M}_{p}$, against the maximum aggregate primary arrival rate $\lambda_{s}=\sum_{i} \lambda_{i}^{s}$, for $i \in \mathcal{M}_{s}$.

Fig. 4 depicts the stability region of the system composed of the primary, relays, and secondary nodes. The system has $M_{p}=20$ primary nodes, $M_{r}=10$ relay nodes, and $M_{s}=10$ secondary nodes. The benefits of cooperation for both primary and secondary networks are significant as illustrated. For instance, at $\lambda_{p}=0.2$ we observe a $350 \%$ increase in the secondary throughput. Moreover, it is noted that both networks benefit from increasing the fraction of idle time slots assigned to relays for cooperation. On one hand, the primary network benefits from that increase since it will get better service from relays, which in turn increases primary nodes service rates, thus, the network can have an extended stability region by sustaining higher arrival rates. On the other hand, secondary nodes benefit from assigning a higher fraction of idle resources to relays, since this results in both primary nodes and relays having higher service rates, thus, higher probability of empty queues. With higher probability of empty queues, secondary nodes will have an increased number of idle time slots to transmit their packets. So in conclusion, under the current scenario of no interference between relays and secondary nodes, it is beneficial to both primary and secondary networks to assign all idle resource to cooperation.

Fig. 5 depicts the stability region of the system comprised of the primary, relays and secondary nodes queues for $\alpha_{r}=1$. It is clear that increasing the number of relays in the network leads to significant improvement in the overall stability region and not only affects primary nodes stability; e.g., for $\lambda_{p}=$ 0.25 we see around $300 \%$ increase in secondary throughput when using 10 or 15 relays. This is due to the fact that although it is apparent that increasing the number of relays will use more and more of the idle time slots and hence decrease secondary nodes' chance to access the channel, the relays help primary nodes empty their queues at a faster rate, and hence provide the secondary nodes with more opportunities to access the channel. We conclude that, with the secondary nodes able to detect both primary and relay transmissions, it is

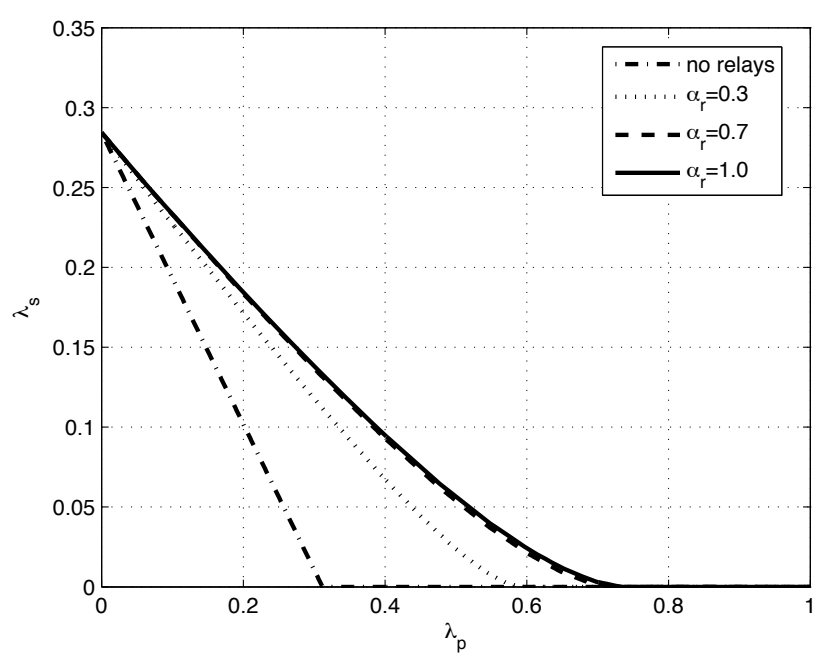

Fig. 6. System stability region with and without cooperation in case of colliding relay and secondary transmissions for different values of $\alpha_{r}$.

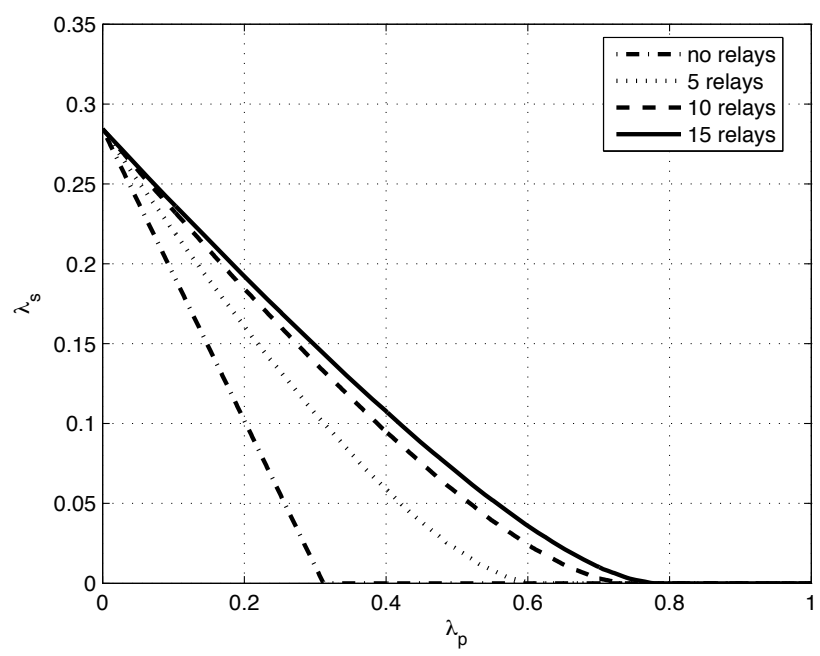

Fig. 7. System stability region with and without cooperation in case of colliding relay and secondary transmissions for different number of relays.

always better to have the maximum number possible of relays, and assign all free resources to cooperation since this will maximize both primary and secondary networks throughput.

Finally, we consider the worst case scenario in which secondary nodes cannot detect relay transmissions, hence always colliding with them. Fig. 6 depicts the stability region for a system with $M_{p}=20$ primary users, $M_{s}=10$ secondary users, and $M_{r}=10$ relays nodes, for different values of $\alpha_{r}$, and for different values of relays in Fig. 7. Despite the fact that relay and secondary nodes are competing for idle channel resources, significant improvements in the stability regions of both primary and secondary network are observed. It is noted that, as in the case without collisions, both networks benefit from assigning more resources for cooperation, or increasing the number of relays in the primary network, although this increase is apparently increasing the probability of collision. This is mainly because the gains of cooperation on the service rates of (27), (29),(32), and (34) exceed the degradation caused 
by collisions. These gains are even more significant for higher primary arrival rate, where secondary nodes achieve much higher throughput even in the case of increased interference.

\section{CONCLUSIONS}

In this paper, the exploitation of idle channel resources in a TDMA network is investigated. First, the use of these idle channel resources cognitively by a group of relay nodes that help the source nodes is studied. Two different relay selection criteria, namely, the nearest neighbor and maximum success probability, were proposed and analyzed. Stability analysis reveals that the cognitive relays can lead to up to $167 \%$ increase in the maximum stable throughput of the network. Then the problem of sharing the idle channel resources between the group of relays and a group of secondary nodes trying to transmit new information over the network is considered. Two different scenarios are studied in details, the first is when the secondary nodes can sense relay transmissions and organize their access to the channel accordingly, the second scenario is when the secondary nodes interfere with relays transmissions in the idle time slots. These two scenarios form inner and outer bounds on the actual network's maximum stable throughput region. Numerical results reveal that under both scenarios, it is beneficial to both the primary and secondary networks that the maximum number possible of relays is always used. That is because the gain to both networks due to cooperation outweighs the losses that might occur due to the interference between relays and secondary nodes transmissions.

\section{APPENDIX \\ NeCESSARY AND SUfFICIENT CONDITIONS FOR STABILITY}

Given identical initial queue sizes for both the original and dominant systems, secondary nodes queues in the dominant system are never shorter than those in the original one. This is true because in the dominant system, secondary nodes suffer from an increased collision probability, thus longer queues, compared to the original one since secondary nodes always have a packet to transmit (possibly a dummy packet). This implies that relay nodes' queues empty faster in the original system and therefore relays see a lower probability of collision as compared to the dominant system, and as a result will have shorter queues. Consequently, stability conditions for the dominant system are sufficient for the stability of the original system.

To prove the necessary conditions, we follow an argument similar to that used by [13] for ALOHA systems to prove the "indistinguishability" of the dominant and original systems at saturation. Consider the dominant system in which secondary nodes transmit dummy packets. If along some realizations of secondary queues of nonzero probability, secondary queues never empty, then the original system and the dominant system are "indistinguishable". Thus, with a particular initial condition, if secondary queues in the dominant system never empty with nonzero probability (i.e., it is unstable), then secondary queues in the original system must be unstable as well. This means that the boundary of the stability region of the dominant system is also a boundary for the stability region of the original system. Thus, conditions for stability of the dominant system are sufficient and necessary for the stability of the original system.

\section{REFERENCES}

[1] J. N. Laneman, D. N. C. Tse, , and G. W. Wornell, "Cooperative Diversity in Wireless Networks: Efficient Protocols and Outage Behavior," IEEE Trans. Inf. Theory, vol. 50, pp. 3062-3080, Dec. 2004.

[2] A. K. Sadek, W. Su, and K. J. R. Liu, "Multinode Cooperative Communications in Wireless Networks," IEEE Trans. Signal Proc., vol. 55, no. 1, pp. 341-355, Jan. 2007.

[3] K. J. R. Liu, A. K. Sadek, W. Su, and A. Kwasinski, Cooperative Communications and Networking, Cambridge University Press, 2008.

[4] A. K. Sadek, K. J. R. Liu, and A. Epheremides, "Cognitive Multiple Access via Cooperation: Protocol Design and Performance Analysis," IEEE Trans. Inf. Theory, vol. 53, no. 10, pp. 3677-3696, Oct. 2007.

[5] S. Haykin, "Cognitive Radio: Brain-Empowered Wireless Communications," IEEE J. Sel. Areas Commun., vol. 23, no. 2, pp. 201-220, Feb. 2005.

[6] N. Devroye, P. Mitran, and V. Tarokh, "Achievable Rates in Cognitive Radio," IEEE Trans. Inf. Theory, vol. 52, no. 5, pp. 1813-1827, May 2006.

[7] A. Jovicic and P. Viswanath, "Cognitive Radio: An InformationTheoretic Perspective," in Proc. IEEE Intl. Symposiom on Infor. Theory (ISIT), Seattle, WA, July 2006, pp. 2413-2417.

[8] Q. Zhao, L. Tong, A. Swami, and Y. Chen, "Decentralized Cognitive MAC for Opportunistic Spectrum Access in Ad Hoc Networks: A POMDP Framework," IEEE J. Sel. Areas Commun., vol. 25, pp. 589600, Apr. 2007.

[9] Y. Xing, R. Chandramouli, S. Mangold, and S. Shankar N., "Dynamic Spectrum Access in Open Spectrum Wireless Networks," IEEE J. Sel. Areas Commun., vol. 24, no. 3, pp. 626-637, Mar. 2006.

[10] N. Benvenuto, S. Pupolin, and G. Guidotti, "Performance Evaluation of Multiple Access Spread Spectrum Systems in the Presence of Interference," IEEE Trans. Veh. Technol., vol. 37, no. 2, pp. 73-77, 1988.

[11] M. Kobayashi, G. Caire, and D. Gesbert, "Impact of Multiple Transmit Antennas in a Queued SDMS/TDMA Downlink," in Proc. 6th IEEE Workshop on Signal Proc. Advances in Wireless Comm. (SPAWC), New York, NY, June 2005.

[12] W. Szpankowski, "Stability Conditions for Some Multiqueue Distributed System: Buffered Random Access Systems," Adv. Appl. Probab., vol. 26, pp. 498-515, 1994.

[13] R. Rao and A. Ephremides, "On the Stability of Interacting Queues in a Multi-Access System," IEEE Trans. Inf. Theory, vol. 34, pp. 918-930, Sept. 1988

[14] R. M. Loynes, "The Stability of a Queue with Non-Independent Interarrival and Service Times," Proc. Cambridge Philos. Soc., pp. 497520, 1962.

[15] D. Cabric, S. M. Mishra, and R. W. Brodersen, "Implementation Issues in Spectrum Sensing for Cognitive Radio," in Proc. Asilomar Conf. on Signals, Syst., and Comput., Pacific Grove, CA, 2004, pp. 772-776.

[16] D. Bertsekas and R. Gallager, Data Networks, Prentice Hall, 2nd edition, 1992.

[17] A. K. Sadek, Z. Han, and K.J.R. Liu, "Distributed relay-assignment protocols for coverage extension in cooperative wireless networks," IEEE Trans. Mobile Computing, vol. 9', number=.

[18] W. Luo and A. Ephremides, "Stability of N Interacting Queues in Random-Access Systems," IEEE Trans. Inf. Theory, vol. 45, no. 5, pp. 1579-1587, July 1999 .

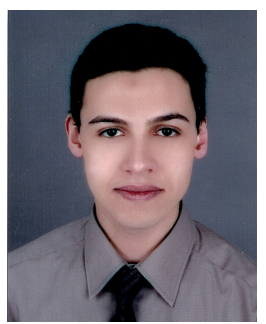

Amr El-Sherif (S' 00, M' 08) received the B.Sc. degree (with highest Honors) and the M.Sc. degree in electrical engineering form Alexandria University, Alexandria, Egypt in 2002 and 2005, respectively. $\mathrm{He}$ received the Ph.D. degree in electrical engineering from the University of Maryland, College Park, in 2009.

He is currently an Assistant Professor in the Electrical Engineering Department at Alexandria University, Egypt. His research interests include cooperative communications and networking, crosslayer design for wireless networks, multiple access technologies for wireless and sensor networks, and spectrum sharing and cognitive radio systems. 


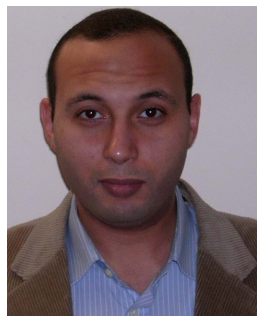

Ahmed K. Sadek (M'07) received the B.Sc. degree (with highest Honors) and the M.Sc. degree in electrical engineering from Alexandria University, Alexandria, Egypt in 2000 and 2003, respectively. $\mathrm{He}$ received the Ph.D. degree in electrical engineering from the University of Maryland, College Park, in 2007. He is currently a Senior Engineer with Corporate Research and Development, Qualcomm Incorporated. His current research interests are in the areas of cognitive radios, spectrum sharing, interference management, cooperative communications, and

MIMO-OFDM systems.

In 2000, Dr. Sadek won the first prize in IEEE Egypt Section undergraduate student contest for his B.Sc. graduation project. He received the Graduate School Fellowship from the University of Maryland in 2003 and 2004, and the Distinguished Dissertation Fellowship award from the Department of Electrical Engineering, University of Maryland in 2007. He is a co-author of Cooperative Communications and Networking, Cambridge University Press, 2008.

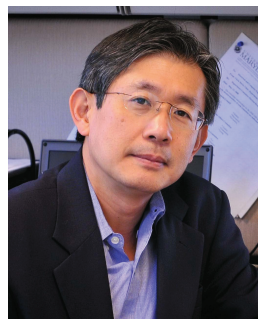

K. J. Ray Liu (F'03) is named a Distinguished Scholar-Teacher of University of Maryland, College Park, in 2007, where he is Christine Kim Eminent Professor of Information Technology. He is Associate Chair of Graduate Studies and Research of Electrical and Computer Engineering Department and leads the Maryland Signals and Information Group conducting research encompassing broad aspects of wireless communications and networking, information forensics and security, multimedia signal processing, and biomedical engineering.

Dr. Liu is the recipient of numerous honors and awards including IEEE Signal Processing Society Technical Achievement Award and Distinguished Lecturer. He also received various teaching and research recognitions from University of Maryland including university-level Invention of the Year Award; and Poole and Kent Senior Faculty Teaching Award and Outstanding Faculty Research Award, both from A. James Clark School of Engineering. An ISI Highly Cited Author in Computer Science, Dr. Liu is a Fellow of IEEE and AAAS.

Dr. Liu is President-Elect and was Vice President - Publications of IEEE Signal Processing Society. He was the Editor-in-Chief of IEEE Signal Processing Magazine and the founding Editor-in-Chief of EURASIP Journal on Advances in Signal Processing.

His recent books include Cognitive Radio Networking and Security: A Game Theoretical View, Cambridge University Press, 2010; Behavior Dynamics in Media-Sharing Social Networks, Cambridge University Press (to appear); Handbook on Array Processing and Sensor Networks, IEEE-Wiley, 2009; Cooperative Communications and Networking, Cambridge University Press, 2008; Resource Allocation for Wireless Networks: Basics, Techniques, and Applications, Cambridge University Press, 2008; Ultra-Wideband Communication Systems: The Multiband OFDM Approach, IEEE-Wiley, 2007; Network-Aware Security for Group Communications, Springer, 2007; Multimedia Fingerprinting Forensics for Traitor Tracing, Hindawi, 2005. 